\title{
Inaugural Editorial: Food Digestion
}

\author{
Roger Lentle
}

Published online: 24 November 2010

(C) Springer Science+Business Media, LLC 2010

The launching of a new journal is said to signal the maturity of a scientific field. In the case of the science of food digestion, this maturity is derived principally from the consolidation of general and scientific interest in the field and from its practical relevance, rather than from the genesis of new methodologies. The science of nutrition has been popularised by findings that relate the quantitative intake of various nutrients to the enhancement of health or to the onset of disease. The attendant upsurge of interest in the processes and dynamics by which food is digested largely relates to ensuring that these nutrients are digested and absorbed and delivered in appropriate quantities.

Two recent developments have boosted scientific research in the process of digestion and absorption. The first relates to the effects of small quantities of substances in foods that can have beneficial effects on health over and above their values as nutrients, i.e. neutraceuticals. The advent of regulations requiring that claims for nutraceutical effects should be based on sound scientific evidence has posed problems in experimental design. A consistent neutraceutical effect can only be assured if the 'dose' of the beneficial agent is consistently delivered and absorbed rather than degraded, excreted or otherwise rendered nonavailable. In a number of cases, the quantity of the neutraceutical agent contained in a given food is low and the dose and form required to elicit the reported effect is unknown. A particular case is the microbial processing, deglycosylation, derivitisation and absorption of plant pigments in the colon.

\section{R. Lentle $(\square)$}

Institute of Food,

Nutrition and Human Health, Massey University,

Private Bag 11222,

Palmerston North, New Zealand

e-mail: r.g.lentle@massey.ac.nz
The second recent development relates to the upsurge of interest in and popularity of methods that assess the relative rates at which macronutrients enter the systemic circulation after the administration of doses of food and relate this to medical consequences. The glycaemic index is the foremost of these. Whilst these tests may be useful in assessing the overall effect of a given food formulation, they can provide little insight into the manner in which the various physical, physiological and chemical processes of digestion are influenced to produce this outcome. There is a need for more specific and more readily reproducible assays of specific digestive processes which can be used to explore the effects of a particular means of food processing so as to better achieve a given outcome. A number of in vitro and in silico systems have been developed that attempt to simulate particular digestive processes, in particular, human digestive organs. Whilst these systems have greater reproducibility and a degree of utility, their fidelity again depends on a detailed knowledge of the processes that they attempt to recapitulate.

If we are to develop high-fidelity tests with which to assay the effects of food processing and formulation of macronutrients or micronutrients and to manipulate these to beneficial ends, we need relevant and robust understanding of the component processes of food digestion. The new journal Food Digestion aims to provide a forum for publication of research dedicated to obtaining such information as well as serving as a source of reference and communication. Hence, Food Digestion will publish papers on an international basis from a broad range of relevant sciences that pertain to the physical, chemical, biochemical, physiological and microbiological dynamics of the digestion and absorption of foods, specific nutrients and other agents. We will also publish timely reviews regarding specific aspects of this broad prescription. It is noteworthy that we will accept research communications describing 
digestive and absorptive outcomes for particular nutrients or substances from particular food matrices and work comparing the effects of different matrices on such outcomes, as we are mindful that such work is relevant to the development of appropriate food technologies. Similarly, we will accept manuscripts that describe interactions between and digestive actions of species of commensal enteral microbiota in relation to the intake, digestion and absorption of nutrients.
We recognise that the success of this journal depends on the quality and scientific merit of papers that are published therein. We aim to publish papers of high quality and to maintain a high level of professional integrity in assessment and publication. We have appointed an editorial board of prestigious and reputable members who are prepared to commit their time and intellect to this end. I cordially invite you to use this journal as an outlet for your scientific endeavours. 